\title{
Ionization dynamics in expanding clusters studied by XUV pump-probe spectroscopy
}

\author{
M Krikunova ${ }^{1}$, M Adolph ${ }^{1}$, T Gorkhover ${ }^{1}$, D Rupp ${ }^{1}, \mathrm{~S}$ \\ Schorb $^{1,2}$, C Bostedt ${ }^{2}$, S Roling ${ }^{3}$, B Siemer ${ }^{3}, \mathbf{R}$ Mitzner $^{3,4}, \mathbf{H}$ \\ Zacharias $^{3}$ and T Möller ${ }^{1}$ \\ ${ }^{1}$ Institut für Optik und Atomare Physik, Technische Universität Berlin, \\ Hardenbergstr. 36, EW 3-1, 10623 Berlin, Germany \\ 2 SLAC, National Accelerator Laboratory, P.O. Box 20450, Stanford, California \\ 94309, USA \\ 3 Physikalisches Institut, Westfälische Wilhelms-Universität, Wilhelm Klemm Str. \\ 10, 48149 Münster, Germany \\ 4 Helmholz-Zentrum Berlin für Materialien und Energie, Hahn-Meitner-Platz 1, \\ 14109 Berlin, Germany \\ E-mail: maria.krikunova@physik.tu-berlin.de
}

\begin{abstract}
The expansion and disintegration dynamics of xenon clusters initiated by the ionization with femtosecond soft-X-ray (XUV) pulses were studied with pumpprobe spectroscopy using the autocorrelator setup of the Free-electron LASer facility at Hamburg (FLASH). The ionization by the first XUV pulse of $92 \mathrm{eV}$ photon energy $\left(8 \times 10^{12} \mathrm{~W} / \mathrm{cm}^{2}\right)$ leads to the generation of a large number of quasi-free electrons trapped by the space charge of the cluster ions. A temporally delayed, more intense probe $\left(4 \times 10^{13} \mathrm{~W} / \mathrm{cm}^{2}\right)$ pulse substantially increases a population of nanoplasma electrons providing a way of probing plasma states in the expanding cluster by tracing the average charge of fragment ions. The results of the study reveal a time scale for cluster expansion and disintegration, which depends essentially on the initial cluster size. The average charge state of fragment ions and thus the cluster plasma changes significantly on a time scale of 1-3 ps.
\end{abstract}

PACS numbers: 36.40.Qv , 41.60.Cr, 52.50.Jm, 79.20.Ws, 78.47.J 


\section{Introduction}

Within femtoseconds, intense light pulses are able to transform matter into a highly excited non-equilibrium state. Clusters, as nanometer-sized particles with the density of bulk solids isolated in free space, are ideal to study light-matter interaction and to develop our understanding of the underlying physics [1]. The results of recent studies reveal a strong dependence of the ionization and disintegration dynamics of clusters on the characteristic parameters of the ionizing radiation such as frequency, power density and pulse duration, as well as on the chemical composition and cluster size. In the near-infrared (NIR) spectral region the interaction between strong laser pulses and clusters composed of atoms and/or molecules leads to the production of highly charged energetic ions [2], hot electrons [3, 4], to the emission of x-ray photons, with a characteristic energy of inner-shell ionization of atoms [5,6], or even neutrons [7]. In this photon energy range the response is governed by a nanoplasma, which is formed by the field ionization and is effectively heated by collisional interactions of quasi-free electrons (inverse Bremsstrahlung) as well as by resonant collective excitations (plasmons).

The new generation of accelerator-based light sources, such as free-electron-lasers (FEL's) for the soft-X-ray (FLASH) and hard-X-ray range (LCLS), currently produce coherent high intense laser pulses extending the frontier of studies of nonlinear processes in condensed matter into the x-ray energy domain. Experiments at $100 \mathrm{~nm}$ [8] revealed surprisingly high final charge states of fragment ions indicating enhanced energy absorption in clusters compared to atoms. It turned out, that the collective plasma heating effects in the vacuum ultraviolet spectral region (VUV) at $100 \mathrm{~nm}$ are still quite large, even though the amplitude of free-electron motion in an oscillating field (quiver amplitude) becomes negligible. Photoelectron spectra confirmed a thermal kinetic energy distribution [9]. According to theoretical work [10,11] the heating of quasi free electrons in the VUV spectral region occurs preferably via inverse Bremsstrahlung and through many body collisions. Within the extreme ultraviolet and soft x-ray region (XUV) the ionization dynamics is controlled by the atomic absorption crosssection $[12,13]$. Here the nanoplasma heating becomes less efficient for $\mathrm{Ar}$ and $\mathrm{Kr}$ clusters $[12,14]$, while for Xe cluster at $90 \mathrm{eV}$ photon energy the nanoplasma is again effectively heated [15] by the electronic absorption and energy exchanging collisions of trapped photo and Auger electrons.

The understanding of nanoplasma dynamics requires considerable theoretical and experimental efforts. Many detailed studies were undertaken to access the temporal evolution of the cluster response to the ionizing radiation from the NIR to the hard x-ray spectral region $[16,17,18,19]$, revealing a complex interplay between plasma heating effects on the one side and plasma cooling due to charge recombination and hydrodynamic expansion on the other side. A detailed modeling of multielectron processes governing the cluster disintegration is still difficult, because different time scales are involved. Therefore, various experiments for probing the transient plasma states are proposed by several theoretical groups [17, 19]. While time-resolved 


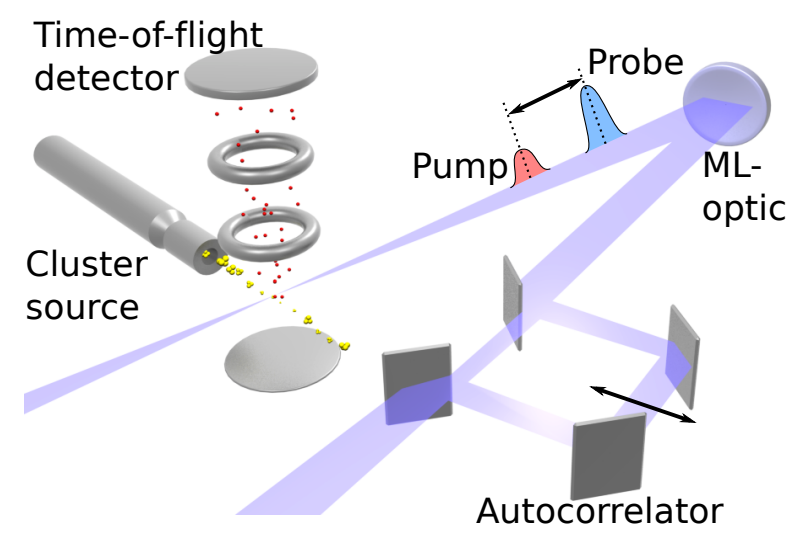

Figure 1. Scheme of the experimental geometry: pump and temporally delayed probe pulses produced by the autocorrelator are focused with the multilayer (ML) mirror on the Xe cluster target. Ions created during the cluster disintegration within the interaction region are detected by a time-of-flight mass spectrometer.

experiments are already performed in the NIR [20], experimental results with short wavelength radiation, however, are collected without any direct time resolution. In these studies final distributions of photoelectrons or ionic fragments are detected long after cluster disintegration is completed $[12,13]$. Nevertheless, recent results $[21,22,23]$ provide experimental evidence that a highly charged cluster surface explodes while in the inner part of the cluster an almost neutral plasma is formed.

In this work, we investigate the ionization dynamics of rare gas clusters in the XUV spectral range using pump-probe techniques. The first XUV pulse $(92 \mathrm{eV})$ with a moderate intensity $\left(8 \times 10^{12} \mathrm{~W} / \mathrm{cm}^{2}\right)$ ionizes Xe atoms within the clusters. A large number of electrons (up to one electron per cluster atom) is released upon photoabsorption from individual atoms inside the cluster due to the dominant ionization of the $4 \mathrm{~d}$ core level of Xe atoms at this photon energy, followed by a single or a cascade of two Auger decay processes [24]. Most of the activated electrons, however, remain quasi-free, i.e., trapped by the Coulomb potential of the cluster [12]. A temporally delayed, more intense probe pulse $\left(4 \times 10^{13} \mathrm{~W} / \mathrm{cm}^{2}\right)$ significantly increases the population of the nanoplasma electrons. At the used photon energy multi-electron collisions are the only mechanism which could be responsible for plasma thermalization and heating [15] leading to a further growth of the cluster Coulomb potential. Finally, upon cluster disintegration, fragment ion spectra are recorded as a function of time delay between pump and probe pulses. As will be discussed in the text, the average charge in the fragment ion distribution is sensitive to the degree of cluster ionization as well as to the cluster radius. Therefore, by monitoring of the average charge of fragment ions as a function of time delay the expansion of the clusters is followed. 


\section{Experiment}

The experiments were performed at beamline BL3 of the FLASH facility operated at a photon energy of $92 \mathrm{eV}$ (13.4 nm wavelength) and $5 \mathrm{~Hz}$ repetition rate in single bunch mode [25]. A scheme of the experimental geometry is shown in Figure 1. The autocorrelator setup, which is especially developed for FLASH [26] produces two timecorrelated soft-X-ray (XUV) pulses. In the present experiment, we have used $20 \%$ intensity within the fixed arm as pump beam and $80 \%$ intensity within the variable arm as time-delayed probe beam. The temporal overlap between pump and probe pulse, which corresponds to zero time delay, was determined by the measurement of the contrast of two-beam interference patterns generated by the overlap of both beams on a soft X-ray sensitive CCD camera as explained in [26]. The spatial overlap between the two beams is crucial for the experiments. It has been controlled carefully between the measurements, since already small movements of the autocorrelator could lead to a reduced overlap.

In the interaction region both beams from the XUV autocorrelator are spatially overlapped and focused by a normal incidence spherical Mo/Si multilayer mirror with a focal length of $450 \mathrm{~mm}$ to a spot size of about $25 \mu \mathrm{m}$ full-width-at-half-maximum (FWHM). For each time delay the spatial overlap was verified by imaging the pump and probe profiles on a fluorescent screen with a microscope. Assuming $35 \mathrm{fs}$ FWHM XUV pulse duration [27, 28] and taking the transmission of the beamline [25], the autocorrelator setup [26] and the multilayer mirror (60\%) into account, the estimated average XUV intensity within the interaction region was in the order of $8 \times 10^{12} \mathrm{~W} / \mathrm{cm}^{2}$ for the pump and of $4 \times 10^{13} \mathrm{~W} / \mathrm{cm}^{2}$ for the probe beams, respectively. The charge states produced in the atomic gas target at these intensities were similar to those observed previously [29].

Although the ion spectra can be sorted according to intensity of the incoming radiation, some fluctuations due to beam displacements in the autocorrelator caused by vibrations could not be avoided. Before taking pump-probe spectra with the different delays, ion spectra were recorded with pump and probe pulse separately. This allows us to proof, whether the experimental conditions for each measured time delay are within statistical fluctuations.

The clusters are produced by adiabatic expansion of Xe gas through a pulsed $100 \mu \mathrm{m}$ conical nozzle with an half-opening angle of $15^{\circ}$. A stagnation pressure of 2.9 and 8 bar at a temperature of $300 \mathrm{~K}$ was applied to prepare pristine clusters comprising of $\langle N>\approx 850$ and of $\langle N>\approx 9500$ atoms, respectively. The cluster size is estimated by using scaling laws given in $[30,31,32]$. Prior to the interaction region the cluster beam was sent through a differentially pumped double skimmer system. The pressure within the main experimental chamber with the cluster source on was $\sim 3 \times 10^{-7}$ mbar. Different charge states of Xe ions created in the target volume were detected by a time-of-flight mass-to-charge spectrometer (TOF spectrometer). An aperture inside the spectrometer has been used to restrict the detection region to the focal volume. 

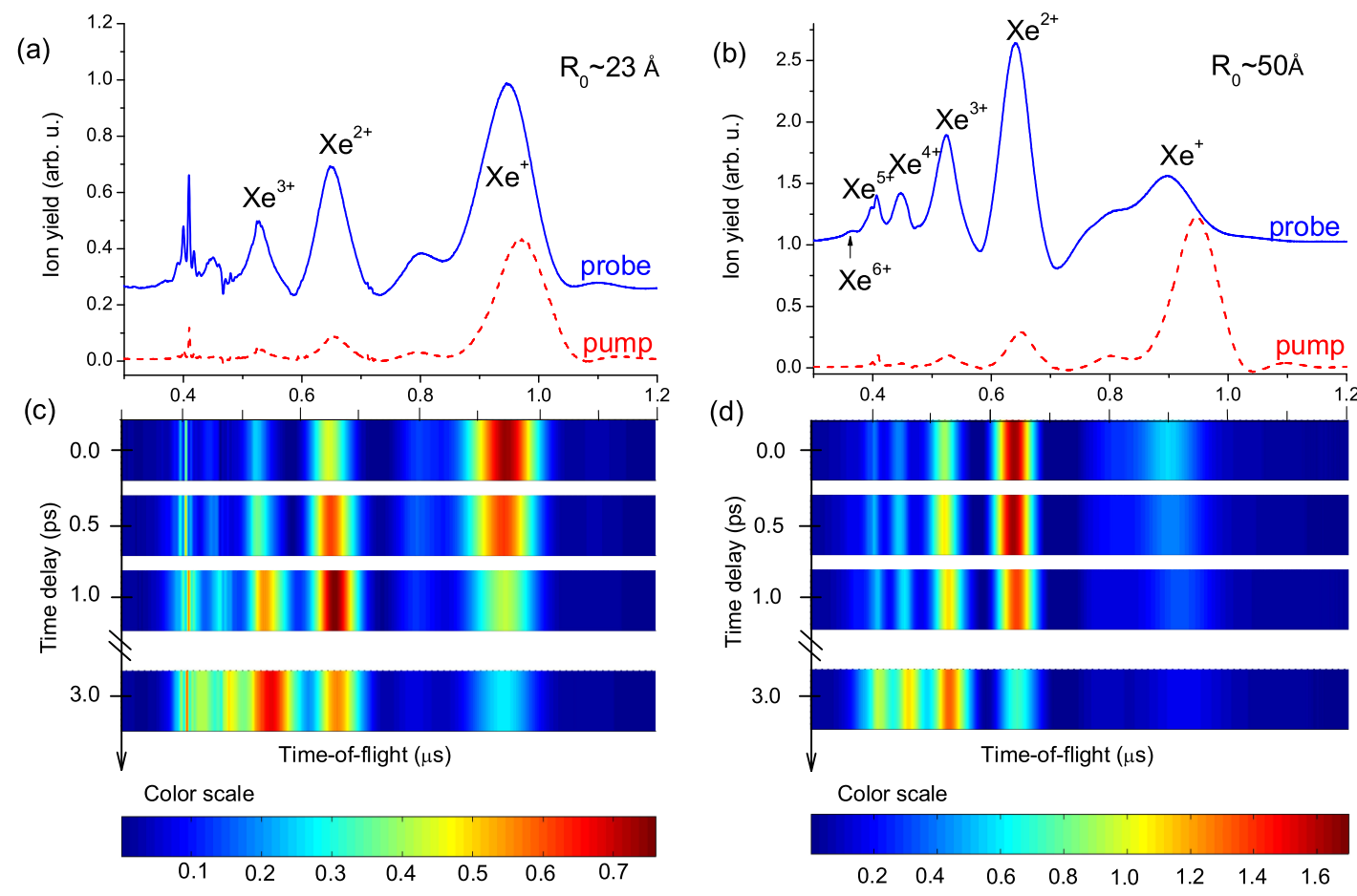

Figure 2. (a) and (b) - Ion yield spectra produced, respectively, by the irradiation of the Xe cluster of initial radius $\mathrm{R}_{0} \sim 23 \AA$ and $\mathrm{R}_{0} \sim 50 \AA$ with exclusively pump (red dashed line) or probe (blue solid line) fields. (c) and (d) color plots plotted on the same color scale shown below - ion yield spectra produced after the interaction of the corresponding cluster with pump and delayed probe XUV pulses. Spectra were measured at 0, 0.5, 1 and 3 ps time delay. Top and bottom spectra are plotted on the same time-of-flight scale. Each trace was typically averaged over 1000 single shots.

\section{Results and discussion}

\subsection{Cluster size and power density dependent features in fragment ion spectra}

The ion yield spectra from Xe clusters composed of $\langle N\rangle \approx 850$ atoms (estimated initial radius $\left.\mathrm{R}_{0} \sim 23 \AA\right)$ and of $\langle N\rangle \approx 9500$ atoms $\left(\mathrm{R}_{0} \sim 50 \AA\right)$ irradiated exclusively by the pump (dashed line) or probe (solid line) pulses are shown in Figure 2 (a) and (b), respectively. The spectra of both cluster sizes produced by the pump pulse alone (Figure 2 (a) and (b) dashed line) are dominated by the $\mathrm{Xe}^{1+}$ charge state. For the free atom, however, mainly $\mathrm{Xe}^{2+}$ and $\mathrm{Xe}^{3+}$ charge states are observed even under weak excitation with synchrotron radiation at $92 \mathrm{eV}$ photon energy, because of preferable inner-shell ionization with subsequent Auger transitions [24]. The lower ionic charge states produced by ionization of clusters compared to free atoms are mostly explained by the large Coulomb potential of the cluster, which prevents electrons to escape the cluster (outer ionization). Furthermore, efficient charge transfer and recombination can take place in clusters [23]. 
The appearance of higher ionic charge states is observed (Figure 2 (a) and (b) solid line) in ion spectra produced by the more intense probe pulse, since multiphoton absorption can be expected. Note, that for small clusters the corresponding spectrum (Figure 2 (a) solid line) is affected by a substantial contribution from the residual gas in the experimental chamber (sharp lines at flight times below $0.5 \mu \mathrm{s}$ ). The charge states of cluster fragment ions are slightly higher for larger clusters (Figures 2 (a) and (b)) in agreement with previous work [21]. This is due to the charge transfer inside the cluster [19, 21, 23], which is especially effective in large clusters. Our findings are also in correspondence with previously reported results [33]. In particular, at the end of the XUV pulse the higher ionic charge states are preferably localized on the cluster outer shell while the inner core composed of nanoplasma electrons and ions remains quasi-neutral. The model has been confirmed experimentally by varying the cluster composition from pristine to doped and Xe core-Ar shell systems [23], by analysis of the kinetic energy distribution of fragment ions $[21,22]$ as well as by theoretical calculations $[18,19]$.

To summarize, in the ion yield spectra shown in Figures 2 (a) and (b) we observe that the contribution of higher charge states is increasing for larger clusters as well as for higher power density. This issue has already been addressed in our previous investigations $[23,21]$ and is mostly explained by the inhomogeneous charge redistribution inside the cluster. In the current study we utilize this sensitivity of the ion yield spectra to the cluster radius as well as to the degree of cluster ionization. Therefore, in the following subsection we calculate an average charge state $\bar{Q}$ of the fragment ions from the ion yield spectra measured as a function of time delay between pump and probe pulses. The cluster expansion dynamics is, thus, traced by following the temporal evolution of an average charge state $\bar{Q}$.

\subsection{Cluster expansion dynamics as revealed from time delay dependent ion yield spectra}

The fragment ion charge state spectra as a function of time delay between pump and probe pulses are shown in Figures 2 (c) and (d) for cluster radii $R_{0} \sim 23 \AA$ and $R_{0} \sim 50 \AA$, respectively. A shift of the ion yield distribution towards higher charge states with increasing time delay is clearly visible. To analyze this behavior we convert the time-offlight scale in the ion yield spectra into the charge state scale for Xe ions and determine an average charge $\bar{Q}$ as a center-of-mass from those spectra. We like to note, that the experimental charge state distribution is affected by charge recombination processes during the cluster expansion and to some extend by the transmission function of the spectrometer. These should to be taken into account when comparing the average charge $\bar{Q}$ estimated from the experimental ion charge state spectra with theoretical predictions [17].

The same time-of-flight range $0.3-1.1 \mu$ s for both $\mathrm{R}_{0} \sim 23 \AA$ and $\mathrm{R}_{0} \sim 50 \AA$ cluster sizes is used for the calculation of $\bar{Q}$. Note, that for small clusters $\bar{Q}$ is probably slightly 
(a)

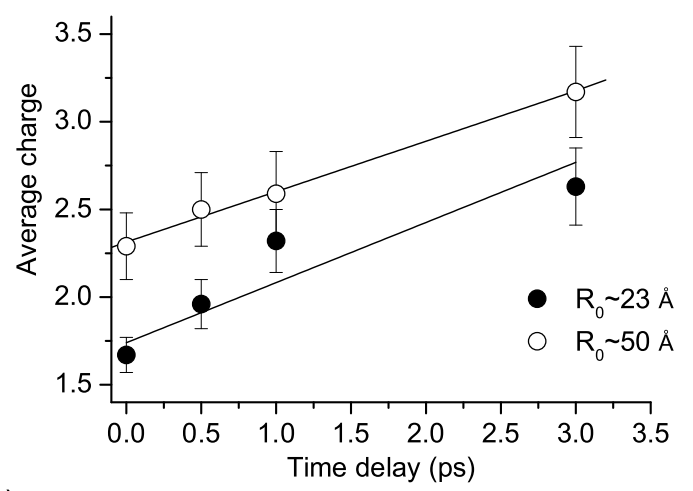

(b)

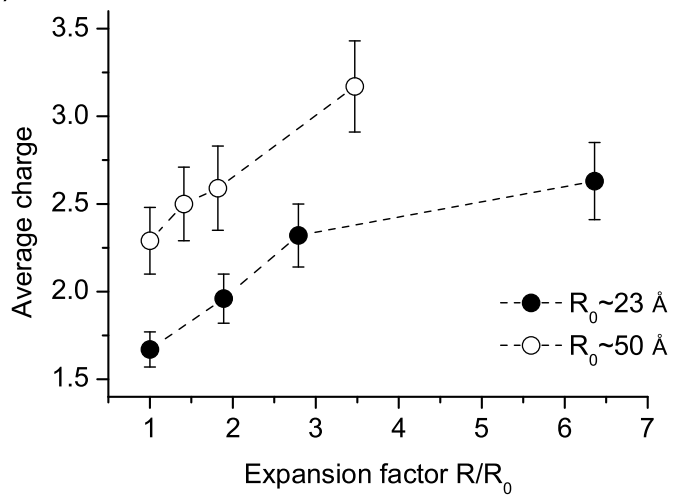

Figure 3. (a) Changes of the average charge as deduced from spectra shown in Figure 2 (c) and (d), respectively, for cluster of radius $\mathrm{R}_{0} \sim 23 \AA$ (closed circles) and $\mathrm{R}_{0} \sim 50 \AA$ (open circles). Lines under the corresponding symbols represent a linear fit to the data. (b) The same data set as shown in (a) on the expansion factor scale (recalculated). Error bars are standard deviations estimated over about 1000 single shot measurements.

overestimated, because of a contribution from the residual gas in the experimental chamber at flight times below $0.5 \mu \mathrm{s}$. According to our estimations the relative changes of $\bar{Q}$ as a function of time delay between pump and probe pulses, however, are only slightly affected by this overestimation. This is because the distribution function of our signal is substantially broader compared to the time delay depended shift of its maximum as well as to the width of an atomic background signal. Also the residual gas contribution does not show a significant dependence on the time delay.

The calculated average charge $\bar{Q}$ as a function of time delay is shown in Figure 3 (a). Each data point represents an average over about 1000 normally distributed single measurements. The increase of the average charge $\bar{Q}$ as a function of time delay is observed for both cluster sizes. We have performed a chi-square test to proof whether the average charge $\bar{Q}$ shows a linear dependence on time delay. The corresponding fits to the data are shown in Figure 3 (a) solid line. The chi-square probability value p for larger $\left(\mathrm{R}_{0} \sim 50 \AA\right)$ clusters is $\mathrm{p}=0.97$ and, thus, a linear function provides an appropriative approximation. For smaller $\left(\mathrm{R}_{0} \sim 23 \AA\right)$ clusters, however, an estimated p value is about 
0.25 , which may probably indicate a saturation effect. Such saturation can be expected for long delay times for all cluster sizes, when the target accessed by the probe pulse is approaching an atomic-like character rather than a cluster as a whole. For Xe atoms no long term (on the ps time scale) dynamics are predicted in theoretical studies [34] and are not observed in time-resolved experiments [35] at this photon energy. Whether the small deviation from the linear fits shown in Figure 3 (a) already indicates some saturation can not unambiguously be decided from our experimental results.

To further analyze the evolution of average charge we consider a model of sonic expansion of a plasma sphere into vacuum. Under the current pump irradiation conditions $\left(8 \times 10^{12} \mathrm{~W} / \mathrm{cm}^{2}\right)$ the number of photons per absorption cross-section estimated for atomic $\mathrm{Xe}$ at $90 \mathrm{eV}$ photon energy is about 0.5. Thus, a rather cold nanoplasma with $\mathrm{Z}=1$ and $\mathrm{T}_{e}=20 \mathrm{eV}$ (approximately the mean kinetic energy of thermalized nanoplasma electrons as deduced from our previous study [15]) is produced. Also, the detection efficiency of our ion spectrometer seems to be higher for slow ions, i.e. with low or without any kinetic energy. This is, particulary, manifested by the decrease of the fast component of the $\mathrm{Xe}^{1+}$ signal in the ion spectra produced by larger clusters at higher power densities (compare $\mathrm{Xe}^{1+}$ signal in Figure 2 (a) and (b) solid line). Hence, our current experimental conditions provide access to the dynamics of rather slow ions, which can be treated in terms of a hydrodynamic expansion model [36].

According to this model a cluster is considered as a sphere expanding with a constant plasma sound speed. A characteristic cluster expansion time (in seconds) can be estimated as discussed e.g. in [36]:

$$
\tau_{\text {exp }}=\left(R-R_{0}\right) \sqrt{\frac{M_{i}}{Z T_{e} \cdot 1.6 \times 10^{-19}}},
$$

where $\mathrm{M}_{i}$ is the mass of the atomic ion (in $\mathrm{kg}$ ), $\mathrm{Z}$ is the mean ion charge, $\mathrm{T}_{e}$ is the electron temperature (in $\mathrm{eV}$ ) and $\mathrm{R}$ is the current cluster radius (in meters). In order to estimate the time needed to double the initial cluster radius from equation (1) we use $\mathrm{Z}=1, \mathrm{~T}_{e}=20 \mathrm{eV}$ as discussed above and $\mathrm{R}=2 \mathrm{R}_{0}$. For smaller clusters $\left(\mathrm{R}_{0} \sim 23 \AA\right)$ this time is considerably shorter $\sim 0.6$ ps compared to $\sim 1.2$ ps for larger clusters $\left(\mathrm{R}_{0} \sim 50 \AA\right)$.

Based on this approach we can now convert the time axis in Figure 3 (a) to the expansion factor $\mathrm{R} / \mathrm{R}_{0}$ as follows:

$$
R / R_{0}=t / R_{0} \sqrt{\frac{Z T_{e} \cdot 1.6 \times 10^{-19}}{M_{i}}}+1
$$

In the present analysis the time delay $\mathrm{t}$ (in seconds) represents the same quantity as the expansion time $\tau_{\text {exp }}$.

Figure 3 (b) shows the almost linear evolution of the average charge with the cluster radius, which is observed for both cluster sizes up to a three to four fold increase of $\mathrm{R} / \mathrm{R}_{0}$. The linear evolution of the average charge, however, is an indication that the Coulomb potential $\mathrm{V}_{\text {Coul }}$ at the cluster surface, determined by a ratio between charge and radius, remains nearly the same during expansion. Indeed, with increasing radius 
of the expanding cluster more nanoplasma electrons can escape the cluster leading to a further accumulation of the positive charge.

For long delay times a charge saturation can be expected. That can be understood in terms of the hydrodynamic expansion model. Assuming, the cluster is composed of spherical shells with an initial interlayer distance of $4.3 \AA$ [37]. The sixfold increase of cluster radius as estimated for smaller $\left(\mathrm{R}_{0} \sim 23 \AA\right)$ clusters at 3 ps time-delay (Figure 3 (b)) would roughly correspond to the interatomic distance of $26 \AA$. Note, the interatomic distance for outermost shells is probably underestimated by this assumption, because these shells expand faster than the inner shells of the cluster [17]. This means, however, that the initial plasma density has declined by more then two orders of magnitude $\left(6^{3}\right.$, see Figure $\left.3(\mathrm{~b})\right)$. At such plasma densities, the cluster Coulomb potential can be considered as essentially flat while interatomic barriers are raised (cf. Figure 3 of [12]).

Thus, we conclude, that the cluster expansion dynamics is manifested by the nearly linear evolution of the average charge as a function of time delay. The probable deviation from this linear behavior may indicate, according to our model, that the clusters are almost completely disintegrated and resemble a strongly diluted plasma.

\section{Summary and outlook}

The expansion dynamics of Xe gas clusters initiated by ionizing XUV radiation is accessed by monitoring the temporal evolution of the average charge in the final fragment ion distribution. The results are understood in terms of the hydrodynamic expansion model. By recalculating the time delay axis to the cluster expansion factor the almost linear evolution of the average charge with the cluster radius is observed for both studied cluster sizes with an up to three to four fold increase of the initial cluster radius. This indicates that the Coulomb potential at the cluster surface, determined by a ratio between charge and radius, remains nearly the same during expansion. The results of the present study show, that the time needed for cluster disintegration increases with an increase of cluster size.

The current approach provides, thus, the possibility to study how the dynamics of spatially restricted nanoplasma is related to its size. The ion yield spectra, however, do not carry information about the electron dynamics and temperature inside the cluster, which is an important parameter for theoretical calculations. Further, the data are integrated over the whole focal volume, i.e., over irradiation intensity profile and cluster size distribution. In future studies the approach should therefore be extended to studies on a single cluster and combined with the measurements of photoelectron and ion spectra simultaneously. This will help to disentangle the dynamics of hot nanoplasma electrons during the cluster expansion. 


\section{Acknowledgments}

This work was supported by BMBF 05KS7KT1, 05KS7KT2 and 05KS4PMC/8 within the priority research program 301 "FLASH", as well as partly by DFG B03169/2-2 and HGFVH VI-302. The authors would like to thank the scientific and technical team of FLASH for machine operation and support at the beamline making this work possible. 


\section{References}

[1] Saalmann U, Siedschlag Ch, Rost JM 2006 Mechanisms of cluster ionization in strong laser pulses J. Phys. B 39 R39

[2] Ditmire T, Tisch J W G, Springate E, Mason M B, Hay N, Smith R A, Marangos J, Hutchinson M H R 1997 High-energy ions produced in explosions of superheated atomic clusters Nature $\mathbf{3 8 6}$ 54

[3] Kumarappan V, Krishnamurthy M, Mathur D 2003 Asymmetric emission of high-energy electrons in the two-dimensional hydrodynamic expansion of large xenon clusters irradiated by intense laser fields Phys. Rev. A 67043204

[4] Springate E, Aseyev S A, Zamith S, Vrakking M J J 2003 Electron kinetic energy measurements from laser irradiation of clusters Phys. Rev. A 68053201

[5] Ditmire T, Donnely T, Falcone R W, Perry M D 1995 Strong X-Ray emission from hightemperature plasmas produced by intense irradiation of clusters Phys. Rev. Lett. 753122

[6] Dobosz S, Lezius M, Schmidt M, Meynadier P, Perdrix M, Normand D 1997 Absolute keV photon yields from ultrashort laser-field-induced hot nanoplasmas Phys. Rev. A 56 R2526

[7] Ditmire T, Zweiback J, Yanovsky V P, Cowan T E, Hays G, Wharton K B 1999 Nuclear fusion from explosions of femtosecond laser-heated deuterium clusters Nature $\mathbf{3 9 8} 489$

[8] Wabnitz $\mathrm{H}$ et al 2002 Multiple ionization of atom clusters by intense soft x-rays from a free electron laser Nature $\mathbf{4 2 0} 482$

[9] Larmann T, Rusek M, Wabnitz H, Schulz J, de Castro A R B, Gürtler P, Laasch W, Möller T 2005 Emission of thermally activated electrons from rare gas clusters irradiated with intense VUV light pulses from a free electron laser Phys. Rev. Lett. 95063402

[10] Santra R, Greene C H 2003 Xenon clusters in intense VUV laser fields Phys. Rev. Lett. 91233401

[11] Siedschlag C, Rost J M 2004 Small rare-gas clusters in soft x-ray pulses Phys. Rev. Lett. 93043402

[12] Bostedt C, Adolph M, Eremina E, Hoener M, Rupp D, Schorb S, Thomas H, de Castro A R B, Möller T 2010 Clusters in intense FLASH pulses: ultrafast ionization dynamics and electron emission studied with spectroscopic and scattering techniques J. Phys. B 43194011

[13] Iwayama $\mathrm{H}$ et al 2009 Frustration of direct photoionization of Ar clusters in intense extreme ultraviolet pulses from a free electron laser J. Phys. B 42134019

[14] Bostedt C, Thomas H, Hoener M, Eremina E, Fennel T, Meiwes-Broer K-H, Wabnitz H, Kuhlmann M, Plönjes, Tiedke K, Treusch R, Feldhaus J, de Castro A R B, Möller T 2008 Multistep ionization of argon clusters in intense femtosecond extreme ultraviolet pulses Phys. Rev. Lett. 100133401

[15] Bostedt C, Thomas H, Hoener M, Möller T, Saalmann U, Georgescu I, Gnodtke C, Rost J-M 2010 Fast electrons from multi-electron dynamics in xenon clusters induced by inner-shell ionization New J. Phys. 12083004

[16] Saalmann U, Rost J M 2002 Ionization of clusters in strong x-ray laser pulses Phys. Rev. Lett. 89 143401

[17] Arbeiter M, Fennel T 2011 Rare-gas clusters in intense VUV, XUV and soft x-ray pulses: signatures of the transition from nanoplasma-driven cluster expansion to Coulomb explosion in ion and electron spectra New J. Phys. 13053022

[18] Ziaja B, Wabnitz H, Weckert E, Möller T 2008 Femtosecond non-equilibrium dynamics of clusters irradiated with short intense VUV pulses New J. Phys. 10043003

[19] Saalmann U 2010 Cluster nanoplasmas in strong FLASH pulses: formation, excitation and relaxation J. Phys. B 430194012

[20] Zweiback J, Ditmire T, Perry M D 1999 Femtosecond time-resolved studies of the dynamics of noble-gas cluster explosions Phys. Rev. A 59 R3166

[21] Thomas H, Bostedt C, Hoener M, Eremina E, Wabnitz H, Laarmann T, Plönjes E, Treusch R, de Castro A R B, Möller T 2009 Shell explosion and core expansion of xenon clusters irradiated with intense femtosecond soft x-ray pulses J. Phys. B $\mathbf{4 2} 134018$ 
[22] Iwayama $\mathrm{H}$ et al 2010 Inhomogeneous charge redistribution in Xe clusters exposed to an intense extreme ultraviolet free electron laser J. Phys. B 43161001

[23] Hoener M, Bostedt C, Thomas H, Landt L, Eremina E, Wabnitz H, Laarmann T, Treusch R, de Castro A R B, Möller T 2008 Charge recombination in soft x-ray laser produced nanoplasmas J. Phys. B 41181001

[24] Holland D M, Codling K, West J B, Marr G V 1979 Multiple photoionization in the rare gases from threshold to $280 \mathrm{eV} \mathrm{J.} \mathrm{Phys.} \mathrm{B.} 122465$

[25] Tiedtke K et al 2009 The soft x-ray free-electron laser FLASH at DESY: beamlines, diagnostics and end-stations New J. Phys. 11023029

[26] Mitzner R et al 2008 Spatio - temporal coherence of free electron laser pulses in the soft x-ray regime Opt. Expr. 16 (24) 19909

[27] Mitzner R et al., 2009 Direct autocorrelation of soft-x-ray free-electron-laser pulses by time-resolved two-photon double ionisation of He Phys. Rev. A 80025402

[28] Frühling U, Wieland M, Gensch M, Gebert Th, Schuette B, Krikunova M, Kalms R, Budzyn F, Grimm O, Rossbach J, Ploenjes E, Drescher M 2009 Single-shot terahertz-field-driven X-ray streak camera Nature Phot. 3523

[29] Sorokin A A, Bobashev S V, Feigl T, Tiedke K, Wabnitz H, Richter M 2007 Photoelectric effect at ultrahigh intensities Phys. Rev. Lett. 99213002

[30] Hagena O F and Obert W 1972 Cluster formation in expanding supersonic jets: effect of pressure, temperature, nozzle size, and test gas J. Chem. Phys. 561793

[31] Buck U, Krohne R 1996 Cluster size determination from diffractive He atom scattering J. Chem. Phys. 1055408

[32] Karnbach R, Joppien M, Stapelfeldt J, Wörmer J, Möller T 1993 CLULU: An experimental setup for luminescence measurements on van der Waals clusters with synchrotron radiation Rev. Sci. Instrum. 642838

[33] Namba S, Hasegawa N, Nishikino M, Kawachi T, Kishimoto M, Sugegawa K, Tanaka M, Ochi Y, Takiyama K, Nagashima K 2007 Enhancement of double Auger decay probability in xenon clusters irradiated with soft-x-ray laser pulse Phys. Rev. Lett. 99043004

[34] Fritzsche S, Grum-Grzhimailo A N, Gryzlova E V, Kabachnik N M 2011 Sequential two-photon double ionization of the $4 \mathrm{~d}$ shell in xenon J. Phys. B 442011

[35] Krikunova $\mathrm{M}$ et al. 2009 Time-resolved ion spectrometry on xenon with the jitter-compensated soft x-ray pulses of a free-electron laser New J. Phys. 11123019

[36] Krainov V P, Smirnov M B 2002 Cluster beams in the super-intense femtosecond laser pulse Phys. Reports 370237

[37] Fugol I Ya 1978 Excitons in rare-gas crystals Adv. Phys. 271 